\title{
You can't cure a disease by throwing money at it, can you?
}

\author{
John W Griffin
}

At the turn of the century (I still love using that phrase), the US Congress supported a doubling of $\mathrm{NIH}$ funds. It is now - understandably - asking what advances in understanding and treatment of major diseases the government investments in neuroscience research have produced. As neurologists, we can sound somewhat defensive when we say, "in addition to spectacular advances in basic neuroscience, we've learned so much about pathogenesis in Alzheimer's and Parkinson's diseases, and rational therapeutic development is underway. Plus, tissue plasminogen activator has been shown to affect stroke outcome-a little." As critics point out, these responses are strong on science, but the major impacts on public health are deferred to the future.

I would nominate HIV dementia as an outstanding example of combined scientific and clinical success resulting from concentrated investment in neuroscience research. Beginning in the mid-1980s, an entirely new neurological disease was recognized and beautifully characterized clinically and pathologically, and within 15 years the main elements of its pathophysiology had been dissected. Research on HIV dementia drove the explosion of interest in microglia and macrophages in neurobiology and in other fields. HIV neuroscience highlighted the roles of cytokines and chemokines in the brain, both in health and disease. It stimulated research on astroglial and cerebral endothelial cell biology. An N-methyl-D-aspartate receptor blocker now used in Alzheimer's disease emerged from studies of the role of glutamate in HIV dementia. HIV neuroscience contributed to the maturation of neuroepidemiology, clinical trial design, and neurocognitive outcome measures. It spawned the development of useful biomarkers of HIV dementia and, more recently, the advent of PET ligands for microglial activation. Skin biopsy techniques used to assess small nerve fiber pathology, now widely used in peripheral nerve research and clinical care (see the Review by Lauria and Devigili in this issue), were developed in part by HIV neuroscientists for research into
"Throwing

money" at

a scientific

problem can

be an effective

strategy, as

long as it is

new money

and not a

destructive

redistribution

of existing

resources

JW Griffin is the Editor-in-Chief of Nature Clinical Practice Neurology.

Competing interests

The author declared no competing interests.

www.nature.com/clinicalpractice doi:10.1038/ncpneuro0629
HIV neuropathy. Finally, neurological prognoses for individuals with HIV have improved dramatically, partly as a result of treating the systemic viral compartment. The problem of CNS disease in HIV is still far from solved, and HIV neuropathy remains an important research target, but it is worth asking why this field has had such signal success overall.

The field of HIV neuroscience enjoyed some distinct advantages in its early days. The level of public and scientific urgency helped to bring superb investigators into the field. As a result of the high death rate in patients with HIV, clinicalneuropathological correlation could be assessed in a fashion not possible in any other disease. Also, neuroscientists had key partnersinfectious disease specialists, retrovirologists and basic immunologists. The challenges, however, including the absence of a small animal model, outweighed these advantages. A key ingredient in the spectacular progress made was the availability of research money.

All of this comes to mind because I heard a colleague complain recently, "Congress is earmarking funds for autism research. They're trying to cure a disease by throwing money at it. The science isn't sufficient to utilize that much money well, and poor studies will be done while good research in other areas goes unfunded." I recall this same argument being made about research in the neuroscience of HIV some 20 years ago. Admittedly, some substandard researchers had funding for one cycle that they might not have received otherwise. In addition, some superb investigators took HIV money and used it to continue studies in their areas of interest, paying only lip service to HIV. I would argue, however, that the HIV research field is a landmark of translational neuroscience, and a success that we should advertise when advocating for adequate funding for diseaseoriented research. "Throwing money" at a scientific problem can be an effective strategy, as long as it is new money and not a destructive redistribution of existing resources. 